\title{
A Rare Cause of Small Bowel Transection: Metastatic Lung Cancer
}

\author{
Mehmet Yildirim $^{a}$ Funda Taslib ${ }^{b}$ M. Emrah Bayam ${ }^{a}$ Hakan Postaci ${ }^{b}$ \\ Departments of a Surgery and ${ }^{b}$ Pathology, Izmir Bozyaka Teaching and Research Hospital, Izmir, Turkey
}

\section{Key Words}

Lung $\cdot$ Small bowel perforation $\cdot$ Metastasis

\begin{abstract}
Objective: To present a case of small bowel perforation due to metastatic lung cancer. Case Presentation and Intervention: A 78-year-old male patient, who had been diagnosed with lung cancer 3 months earlier, presented to our clinic with acute abdominal pain. He underwent emergency laparotomy. At surgery, there was a circumferential lesion encompassing the ileum with complete transection. There was no obvious macroscopic appearance of metastatic disease. The involved bowel segment was resected and an ileostomy was performed. Postoperative pathologic analysis confirmed metastatic squamous cell carcinoma metastasis to the ileum, arising from the patient's lung cancer. Conclusion: This case report showed that small bowel metastases should always be considered in the differential diagnosis of lung cancer patients presenting with acute abdominal pain.

Copyright $\odot 2010$ S. Karger AG, Basel
\end{abstract}

\section{Introduction}

Lung cancer is a leading cause of cancer-related death throughout the world [1]. Approximately one half of patients with lung cancer have metastatic disease at the time of initial diagnosis. The most commonly involved organs are brain, bone, liver, adrenal glands and gastrointestinal tract. Small bowel involvement often leads to perforation, obstruction or bleeding $[2,3]$. Perforations of the small bowel are most often caused by adenocarcinoma; however, metastases from squamous cell and large cell carcinoma are more likely to result in perforation. Symptomatic small bowel metastases from lung carcinoma have rarely been reported [4]. To our knowledge, this is the first case of circumferential small bowel transection due to metastasis.

\section{Case Report}

A 78-year-old male patient who had been diagnosed with lung cancer 3 months earlier presented to our clinic with acute abdominal pain. Prior to hospital admission, he complained of a 1-week history of abdominal pain. He had been evaluated at an oncology hospital, where he had been diagnosed with a $4-\mathrm{cm}$ tumoral mass located in the posterior segment of the left lung. A computed tomography scan of the thorax showed a primary pulmonary tumor invading the pleura, adjacent costa, and multiple enlarged hilar lymph nodes. He underwent a fine-needle biopsy, which disclosed non-small cell lung cancer. Based upon these pathologic and radiologic findings, the patient was considered inoperable. He was treated with a total of 6,600 cGy in 33 sessions of radiotherapy.

Physical examination revealed an afebrile, ill-appearing patient, with a heart rate of $110 \mathrm{bpm}$, a respiratory rate of 28 breaths/ $\mathrm{min}$ and a blood pressure of $100 / 70 \mathrm{~mm} \mathrm{Hg}$. The examination also showed a diffuse abdominal defense and rebound tenderness. Laboratory analyses were within normal limits except for leuko-

\section{KARGER}

Fax +4161306 1234 E-Mail karger@karger.ch www.karger.com
(C) 2010 S. Karger AG, Basel

1011-7571/10/0193-0232\$26.00/0

Accessible online at:

www.karger.com/mpp
Dr. Mehmet Yildirim

Atakent Mah. Bergama 2 Apt. Giris:32 Daire:1

TR-35000 Bostanl1/Izmir (Turkey)

Tel. +90 232362 5692, Fax +90232 2614444

E-Mailmehmetyildi@gmail.com 


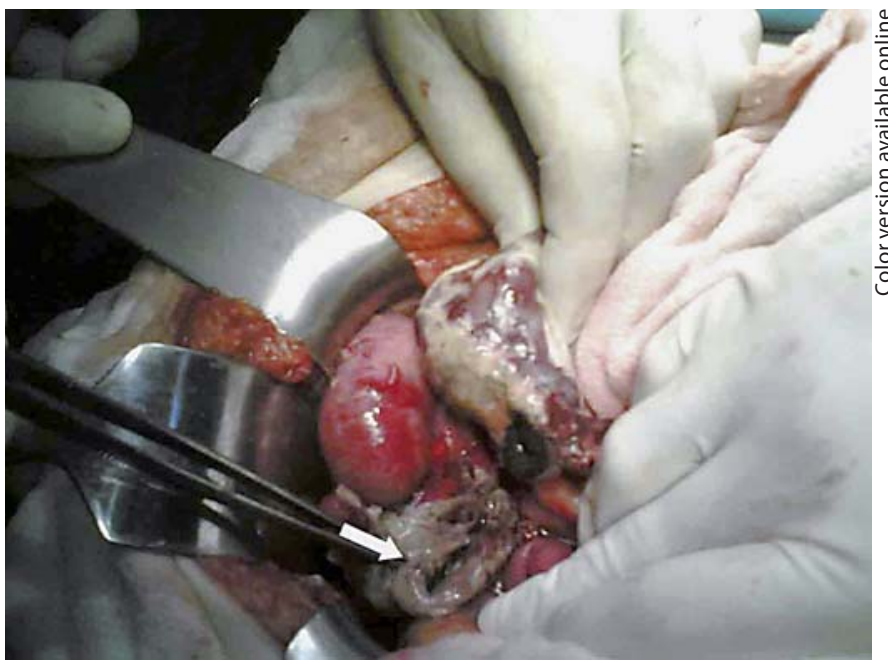

Fig. 1. Operative view of transected intestinal segment (arrow).

cytosis. An abdominal X-ray demonstrated subdiaphragmatic free air.

The patient underwent an exploratory laparotomy, which disclosed a circumferential transection at the terminal ileum; all quadrants had peritonitis with pus and stool (fig. 1). No macroscopically apparent metastatic disease was seen on the transected intestinal wall. Further exploration showed that the remaining abdomen was completely normal. The affected proximal segment of ileum was resected and ileostomy was performed. Histopathologic examination of the specimen revealed an infiltrating tumor in the submucosa, muscularis propria and serosa. No mucosal abnormality was seen in the overlying intestinal mucosa (fig. 2). The lesion consisted of epithelial-like neoplastic cells with significant pleomorphism, large vesicular nuclei, and prominent nucleoli. Extensive single cell keratinization and intercellular bridges between tumor cells were observed (fig. 3). These histological features are characteristic of metastatic squamous cell carcinoma. Postoperative recovery was uneventful and the patient was discharged 7 days after surgery. Two weeks later the patient died from pulmonary failure.

\section{Discussion}

Metastatic lung cancer presenting with small bowel involvement is uncommon. In the literature, 98 cases of small bowel perforation due to metastases from lung cancer have been identified between 1960 and 2005 [2, 5].

Lung cancer metastases spread to the intestines via blood. When the cancer has spread to a small bowel segment, the metastases may be solitary or multiple. Small

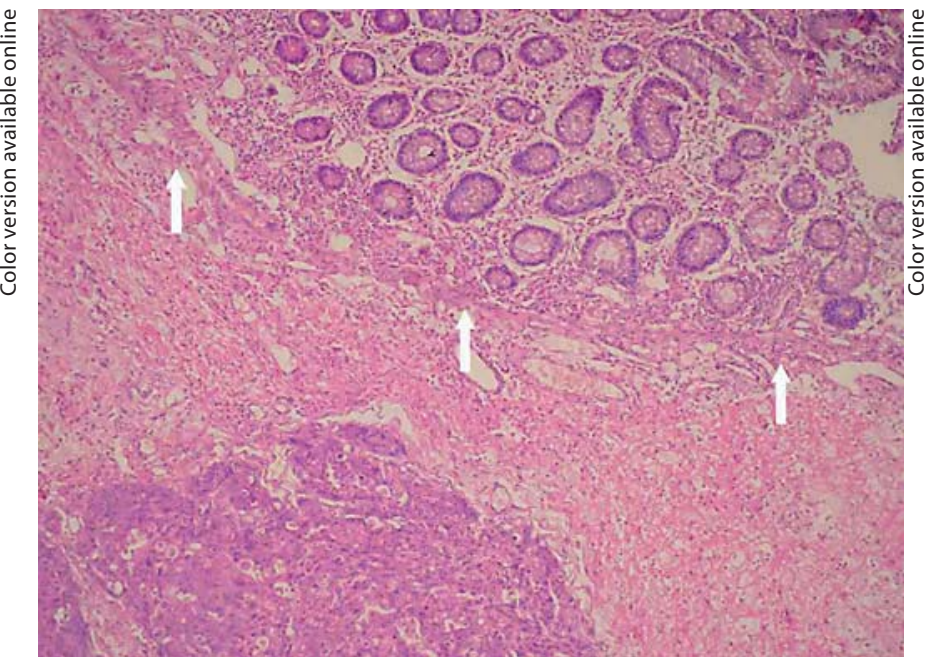

Fig. 2. Histopathologic view of submucosal localization of tumor, separated from the normal mucosa by a preserved lamina muscularis mucosae (arrows). HE. $\times 100$.

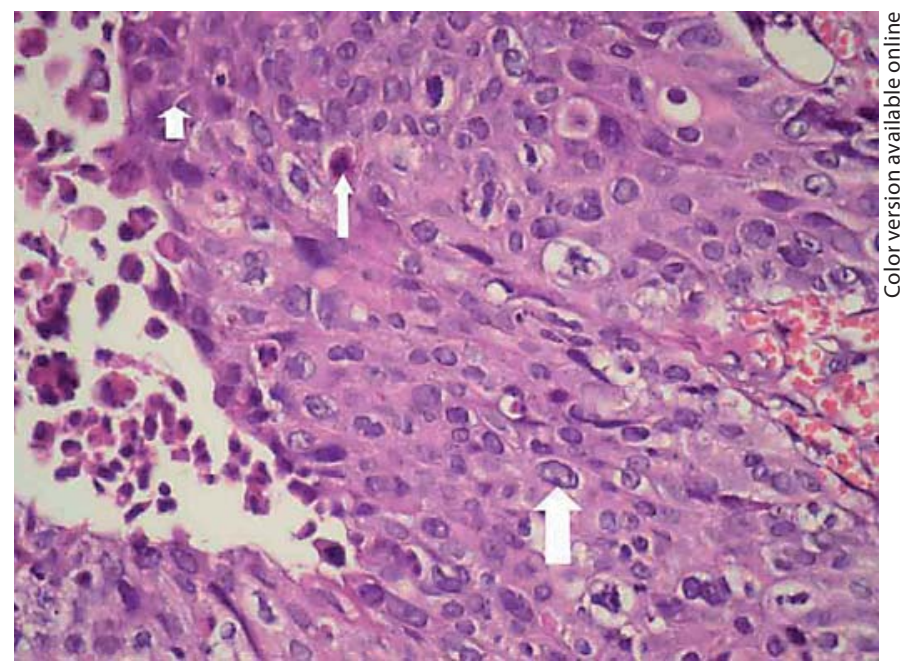

Fig. 3. High-power view of nuclear pleomorphism (large arrow), single-cell keratinization (thin arrow) and intercellular desmosomes (short arrow). HE. $\times 200$.

bowel metastases are located mostly in the jejunum followed by ileum as in our case, and by both sites [4]. Tumor invasion of all or parts of the bowel wall results in necrotic tumor perforation, which may often present as punctured lesions or complete transection of the intestinal wall, which, as in our case, is rare. Metastases are associated with tumors in other sites. In our case, macroscopic disease was not found either at the site of perfora- 
tion or in any other area of the abdomen. Local recurrence is seen more frequently in patients with squamous cell carcinoma, as in our case; patients with adenocarcinoma more often have distant metastases [6].

Acute abdominal pain and other gastrointestinal manifestations are very rare complications of lung cancer. Metastases to the gastrointestinal tract may present with intestinal perforation, luminal obstruction, and gastrointestinal bleeding. Perforation is often preceded by bleeding. Therefore, in a patient with lung cancer, intestinal bleeding may be a predictor of perforation. Screening the small bowel for metastasis cannot be recommended because of its low incidence. However, if a patient with lung cancer presents with intestinal complaints, bowel enema and computed tomography are helpful in diagnosis [1]. Performance status and unclear abdominal complaints cannot provide relevant information on tumor growth in the small bowel. The number of involved organs, perforation sites, and several days' delay may all worsen the patient's status and make surgery more difficult. Mediastinal lymph node involvement indicates an adverse prognosis. In the present case, the advanced stage of the lung cancer and the delayed diagnosis were adverse prognostic factors.
The management of small bowel perforation involves resection of the perforated segment. Metastatic deposits in the perforation site may not be macroscopically apparent, as in our case, and intraoperative findings are insufficient for definitive diagnosis. Despite the fact that surgical resection is technically possible yet unlikely due to comorbid factors, the surgeon's role is usually not curative. Many of these patients are treated primarily by bowel resection. However, there have not been many successful reports of prolonged survival after bowel resection during the past two decades [4].

The use of systemic chemotherapy in non-small cell lung cancer may extend life expectancy but potentially increase the development of distant metastases. Many metastatic tumors are difficult to treat and resistant to therapy. For this reason, palliative therapy and symptomatic control are often needed.

\section{Conclusion}

This case showed that small bowel metastases should be considered in the differential diagnosis of lung cancer patients presenting with acute abdominal pain.

\section{References}

1 Savanis G, Simatos G, Lekka I, Ammari S, Tsikkinis C, Mylonas A, Kafasis E, Nissiotis A: Abdominal metastases from lung cancer resulting in small bowel perforation: report of three case. Tumori 2006;92:185-187.

-2 Sakorafas GH, Pavlakis G, Grigoriadis KD: Small bowel perforation secondary to metastatic lung cancer: a case report and review of the literature. Mt Sinai J Med 2003;70: 130-132.
-3 Galsky M, Darling M, Hecht J, Salgia R: Case 1: small bowel obstruction due to metastatic lung cancer. J Clin Oncol 2000;18:227-228.

4 Yang CJ, Hwang JJ, Kang WY, Chong IW, Wang TH, Sheu CC, Tsai JR, Huang M: Gastro-intestinal metastasis of primary lung carcinoma: clinical presentations and outcome. Lung Cancer 2006;54:319-323.
5 Garwood RA, Sawyer MD, Ledesma EJ, Foley E, Claridge JE: A case and review of bowel perforation secondary to metastatic lung cancer. Am Surg 2005;71:110-116.

6 Huang TW, Wang CH, Tsai WC, Liu YC: Small bowel perforation secondary to metastatic lung cancer: a case report with literature review. Chir Gastroenterol 2006;22:9294. 